\title{
Associations of the BRAF (V600E) mutation and p53 protein expression with clinicopathological features of papillary thyroid carcinomas patients
}

\author{
MI KYUNG SHIN ${ }^{1}$, JEONG WON KIM ${ }^{1}$, SOO KEE MIN ${ }^{4}$, DONG JIN LEE ${ }^{2}$, \\ JIN HWAN KIM ${ }^{2}$, SEUNG CHUL LEE ${ }^{3}$, BONG WHA CHUNG ${ }^{3}$ and YOUNG SU JU ${ }^{5}$
}

\begin{abstract}
${ }^{1}$ Department of Pathology, Kangnam Sacred Heart Hospital, Hallym University Medical Center, Yeongdeungpo-gu, Seoul 150-950; ${ }^{2}$ Department of Pathology, Hallym University Sacred Heart Hospital, Anyang-si, Gyeonggi-do 431-796; Departments of ${ }^{3}$ Otorhinolaryngology-Head and Neck Surgery and ${ }^{4}$ General Surgery, Kangnam Sacred Heart Hospital, Hallym University Medical Center, Yeongdeungpo-gu, Seoul 150-950; ${ }^{5}$ Department of Occupational and Environmental Medicine, Hallym University Sacred Heart Hospital, Anyang-si, Gyeonggi-do 431-796, Republic of Korea
\end{abstract}

Received August 14,2014; Accepted May 20, 2015

DOI: $10.3892 / 01.2015 .3401$

\begin{abstract}
The BRAF (V600E) mutation is the most prevalent type of genetic alteration that has been identified in papillary thyroid carcinoma (PTC); in addition, previous immunohistochemical studies have revealed the overexpression of p53 protein in PTC. The aim of the present study was to investigate the prevalence of the BRAF (V600E) mutation and the expression of p53 in PTC, as well as to determine any associations between these two factors and the clinicopathological features of PTC. The study was performed on 66 PTC patients who underwent surgical tumor resection between January and December 2012. Polymerase chain reaction-based DNA amplification was used to analyze extracted DNA from the tumor specimens in order to determine the prevalence of the BRAF (V600E) mutation. In addition, immunohistochemical analysis was employed in order to evaluate the protein expression of p53 in sections of tumor tissue. Furthermore, statistical analysis was performed in order to determine any associations among the BRAF (V600E) mutation prevalence, p53 overexpression and the clinicopathological features of PTC patients, including age, gender, tumor size, multiplicity, lymph node metastasis and extrathyroidal extension. The results revealed that the BRAF (V600E) mutation was observed in $50(75.8 \%)$ of the 66 PTC patients and overexpression of p53 was found in $52(78.8 \%)$ of 66 cases. No significant correlations were
\end{abstract}

Correspondence to: Professor Mi Kyung Shin, Department of Pathology, Kangnam Sacred Heart Hospital, Hallym University Medical Center, 948-1 Daerim-1Dong, Yeongdeungpo-Gu, Seoul 150-950, Republic of Korea

E-mail: smk8671@naver.com

Key words: BRAF (V600E) mutation, 553 protein, clinicopathologic features, papillary thyroid carcinoma observed between the BRAF (V600E) mutation or p53 protein overexpression and the clinicopathological features of patients. However, the BRAF (V600E) mutation demonstrated noteworthy, but non-significant, correlations with the overexpression of p53 $(\mathrm{P}=0.0854)$ and extrathyroidal extension $(\mathrm{P}=0.0661)$. In addition, a significant correlation was observed between lymph node metastasis and bilaterality $(\mathrm{P}=0.0280)$. In conclusion, the present study demonstrated that the BRAF (V600E) mutation and overexpression of $\mathrm{p} 53$ were not significantly correlated with clinicopathological features of PTC, although notable associations were identified between BRAF (V600E) mutation and overexpression of p53 as well as extrathyroidal extension. In addition, lymph node metastasis was significantly associated with bilaterality.

\section{Introduction}

Papillary thyroid carcinoma (PTC) is a prevalent form of thyroid cancer (1). Due to recent developments in ultrasonography (US) and US-guided fine-needle aspiration biopsies, impalpable small-sized papillary thyroid microcarcinomas have been frequently detected (2).

In general, PTC has a good prognosis; however, certain patients experience local recurrence and/or distant metastasis. Factors that are known to significantly reduce PTC patient prognosis include age, male gender, large tumor size, extrathyroidal extension and metastases (3-6).

Numerous scoring systems for predicting prognosis, including Tumor-Node-Metastases (TNM) and Metastases-Age-Completeness of resection-Invasion-Size of tumor (MACIS) have been used to more accurately establish prognosis in PTC patients $(3,7)$. Furthermore, numerous previous studies have investigated potential molecular and cytological markers of biological behavior (3-7).

The most prevalent type of genetic alteration in PTC is the BRAF (V600E) mutation. BRAF is known to be a mitogen-activated protein kinase (MAPK) signaling pathway activator involved in regulating the growth, division and 
proliferation of cells (8). The V600E amino acid substitution in BRAF is the results of a T1799A point mutation in exon 15 of BRAF; this mutation accounts for $>90 \%$ of all the genetic alterations detected in the BRAF gene (9).

Numerous studies have investigated potential associations among the BRAF (V600E) mutation, clinicopathological features of PTC and the clinical outcome of patients; however, correlations between the incidence of the BRAF (V600E) mutation and clinicopathological features in PTC patients remains controversial (1,9-20).

The incidence rate of the BRAF (V600E) mutation in PTC has been reported to vary between 29 and $83 \%$; of note, the rate of BRAF (V600E) mutation occurrence in Korea was reported to be $52-83 \%$, whereas in other countries the occurrence rate was $30-49 \%$ (9). However, the variation in these results was suggested to be the result of a lack of prospective studies that may reduce the selection bias, small study population sizes, the absence of multivariate analysis and the heterogeneous histological subtypes within PTC (9). Mutations in the tumor suppressor gene p53 were reported to occur in $\sim 50 \%$ of cancers; these mutations account for the most prevalent type of genetic alteration in cancer cells (3-8). Numerous previous studies have demonstrated that genetic mutations of p53 often occur in undifferentiated thyroid cancers. The occurrence of p53 mutations in well differentiated thyroid carcinomas, such as PTC, has not been conclusively established; but the incidence of p53 mutations has been reported as ranging between 0 and 25\% (7,21). p53 protein overexpression, as determine by immunohistochemistry, was reported to be correlated with the presence of p53 gene mutations (7); however, the immunohistochemical detection of p53 overexpression has been identified in differentiated follicular and papillary thyroid carcinomas regardless of the occurrence of p53 gene mutations. p53 protein overexpression was reported to have an incidence rate of between 11 and $59 \%(6,22,23)$. Certain studies regarding the immunohistochemical analysis of $\mathrm{p} 53$ protein expression have demonstrated that p53 overexpression may be used as an independent prognostic indicator for differentiated thyroid carcinomas $(3-7,22,24)$.

The present study aimed to investigate the prevalence of the BRAF (V600E) mutation and the overexpression of $\mathrm{p} 53$ protein in PTC, as well as to determine any potential associations among these two factors and other clinicopathological features of PTC.

\section{Materials and methods}

The present study was approved by the Institutional Review Board of the Kangnam Sacred Heart Hospital of Hallym University Medical Center (no. 2014-04-44; Seoul, Korea).

A total of 66 PTC patients (classic type, 60 cases; follicular variant, 6 cases) who had undergone surgery for the treatment of PTC, thyroid lobectomy or total thyroidectomy with or without lymph node dissection, were enrolled into the present study at the Kangnam Sacred Heart Hospital between January and December 2012. For all the cases, hematoxylin and eosin (H\&E)-stained slides and paraffin blocks for immunohistochemical staining were reviewed. The H\&E slides were examined by two of the present authors, independently, according to the histopathological criteria proposed by the World Health Organization (25) for the diagnosis of PTC. A multi-headed microscope (U-MDOB3, Olympus Corporation, Tokyo, Japan) was used in order to review any slides where the independent reviewers diagnoses were not consistent.

Immunohistochemistry was performed on $4-\mu \mathrm{m}$-thick paraffin-embedded tissue sections using the automated staining system, the Leica Bond-Max autostainer (Leica Microsystems GmbH, Wetzlar, Germany) with appropriate positive and negative controls according to the manufacturer's instructions. Mouse monoclonal anti-p53 antibody (1:3,000; DO-7; Dako, Glostrup, Denmark) was used as the primary antibody. Expression of p53 protein was scored according to intensity and positive cell proportion. The intensity was graded into 0 , none; $1+$, weak; $2+$, intermediate; $3+$; strong. The positive cell proportion was semiquantitatively evaluated according to the estimated percentage of positive tumor cells: 0 , no positive cells; 1 , $<10 \%$ positively-stained cells; $2,0-33 \%$ positively-stained cells; 3, 33-66\% positively-stained cells; $4,>66 \%$ positively-stained cells. The two scores were combined: a total score of $<4+$ was considered negative and scores of $\geq 4+$ were considered positive for $\mathrm{p} 53$.

DNA extraction. Paraffin-embedded tissue was manually micro-dissected into $10 \mu \mathrm{m}$ sections. Genomic DNA was extracted using the QIAamp DNA mini kit (QIAGEN, Chatsworth, CA, USA) according to the manufacturer's instructions.

Analysis of BRAF (V600E) mutation by polymerase chain reaction $(P C R)$. PCR analysis was performed using Seeplex BRAF Autocapillary Electrophoresis Detection kits (Seegene, Seoul, Korea). The PCR reaction mixtures were prepared as follows (total volume, $20 \mu \mathrm{l}$ ): $4 \mu \mathrm{l}$ 5X BRAF primer mix, $3 \mu 1$ extracted DNA (10 ng/ $\mu \mathrm{l}), 3 \mu \mathrm{l}$ 8-methoxypsoralen (8-Mop) solution and $10 \mu \mathrm{l} 2 \mathrm{X}$ multiplex master mix (Seegene). Following incubation at $94^{\circ} \mathrm{C}$ for $15 \mathrm{~min}$, amplification was performed in a 9700 Thermal Cycler (Applied Biosystems, Foster City, CA, USA), with 35 cycles of denaturation at $94^{\circ} \mathrm{C}$ for $30 \mathrm{sec}$, annealing at $63^{\circ} \mathrm{C}$ for $30 \mathrm{sec}$, extension at $72^{\circ} \mathrm{C}$ for $60 \mathrm{sec}$ and a final extension at $72^{\circ} \mathrm{C}$ for $10 \mathrm{~min}$. In order to confirm the presence and size of the amplified PCR products, $5 \mu \mathrm{l}$ was electrophoresed on $2 \%$ (wt/vol) agarose gels containing EtBr (Seegene, Inc., Seoul, Korea): Wild-type BRAF PCR amplification results in a 251-bp amplicon (internal control); BRAF (V600E) results in a 167-bp amplicon (Fig. 1).

For eradicating the template activity of contaminating DNAs, 8-Mop solution was used, which intercalates into double-stranded nucleic acids, forming covalent interstrand cross-links following photoactivation with light of wavelengths 320-400 nm, using a Gel Doc XR+ system (Bio Rad, CA, USA).

Statistical analysis. Values are presented as the mean \pm standard error of the mean. All statistical analyses were performed using SPSS 21.0 software (International Business Machines, Armonk, NY, USA). The Chi-Squared test was used to analyze categorical data and the Student's t-test was used to evaluate continuous variables; all other variables were analyzed using 
Table I. Associations between the BRAF (V600E) mutation and clinicopathological features of papillary thyroid carcinomas.

\begin{tabular}{|c|c|c|c|}
\hline Parameter & BRAF mutation + $(\%)$ & BRAF mutation - $(\%)$ & P-value \\
\hline No. of cases & $50(75.8)$ & $16(24.2)$ & \\
\hline Age, years & $49.5 \pm 10.4$ & $49.3 \pm 10.4$ & 0.9502 \\
\hline Gender & & & 0.7433 \\
\hline Male & $12(70.6)$ & $5(29.4)$ & \\
\hline Female & $38(77.6)$ & $11(22.4)$ & \\
\hline Tumor size (mm) & $9.1 \pm 6.8$ & $7.2 \pm 6.6$ & 0.3272 \\
\hline Multiplicity & & & 1.0000 \\
\hline Present & $19(76.0)$ & $6(24.0)$ & \\
\hline Absent & $31(75.6)$ & $10(24.4)$ & \\
\hline Extrathyroidal-extension & & & 0.0661 \\
\hline Present & $20(90.9)$ & $2(9.1)$ & \\
\hline Absent & $30(68.2)$ & $14(31.8)$ & \\
\hline
\end{tabular}

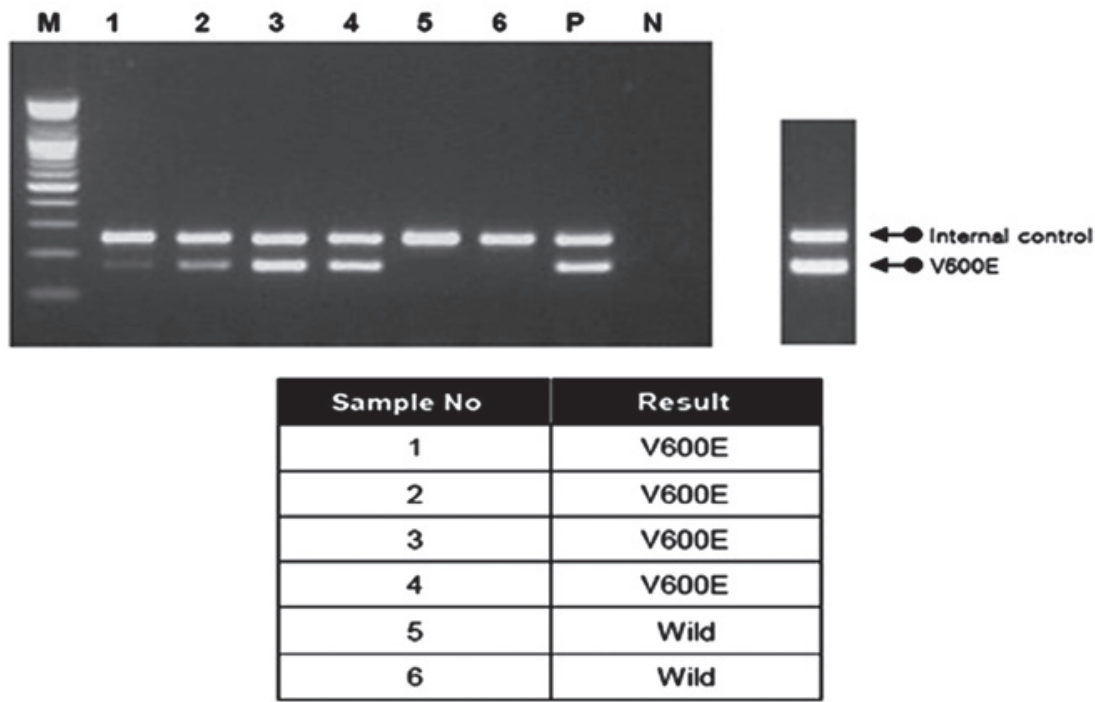

Figure 1. PCR analysis of thyroid specimens. Two clear PCR bands were identified with 251-bp (internal control) and 167-bp (mutant band). Samples 1-4 exhibit the BRAF (V600E) mutation; samples 5 and 6 exhibit wild type BRAF. PCR, polymerase chain reaction; M, 100-bp ladder; P, positive control; N, negative control; 1-6, clinical samples.

Fisher's exact test. $\mathrm{P}<0.05$ was considered to indicate a statistically significant difference.

\section{Results}

Clinicopathological features, BRAF (V600E) status and p53 protein status in PTC. A total of 66 patients were enrolled in the present study, with 17 males $(25.8 \%)$ and 49 females $(74.2 \%)$, with a mean age of $49.5 \pm 10.3$ years (range, 31-74 years) at the time of surgery. A total of 58 patients $(87.9 \%)$ underwent a total thyroidectomy with or without neck dissection. Of these 58 patients, 2 patients $(3.0 \%)$ underwent concurrent comprehensive neck dissection [level II-VI (26)], 2 patients underwent level IV lymph node dissection, 2 patients underwent level II and level III lymph node dissection, 1 patient underwent level I and level II lymph node dissection, 1 patient underwent level II and central lymph dissection, 1 patient underwent level IV and central lymph node dissection and 35 patients $(53.0 \%)$ underwent central neck dissection (CND). In addition, 7 patients (10.6\%) underwent lobectomy with (2 patients) or without (5 patients) CND and 1 patient underwent subtotal thyroidectomy without lymph node dissection.

Among the 66 cases, 55 cases $(83.3 \%)$ were diagnosed as microcarcinoma and the mean tumor size was $8.7 \mathrm{~mm} \pm 6.7$ (range, 0.1-4.0 cm). 60 cases were classic type and 6 cases were follicular variant. One of the $6 \approx$ follicular variant cases was encapsulated follicular variant and the remaining 5 cases were diffuse follicular variant. BRAF (V600E) was identified in 50 cases $(75.8 \%)$ and p53 overexpression was detected in 52 cases $(78.8 \%)$ (Tables I and II).

Associations between BRAF (V600E) status and clinicopathological features of PTC. As shown in Table I, among the 50 patients with the BRAF (V600E) mutation, 12 were males $(24 \%)$ and 38 were females $(76 \%)$, with a mean age of 
Table II. Associations between p53 protein overexpression and clinicopathological features of papillary thyroid carcinomas.

\begin{tabular}{|c|c|c|c|}
\hline Parameter & p53 overexpression + $(\%)$ & p53 overexpression - (\%) & P-value \\
\hline No. of cases & $52(78.8)$ & $14(21.2)$ & \\
\hline Age (years) & $49.7 \pm 10.1$ & $48.6 \pm 11.5$ & 0.7217 \\
\hline Gender & & & 1.0000 \\
\hline Male & $14(82.4)$ & $3(17.6)$ & \\
\hline Female & $38(77.6)$ & $11(22.4)$ & \\
\hline Tumor size (mm) & $9.1 \pm 6.7$ & $7.0 \pm 6.9$ & 0.3007 \\
\hline Multiplicity & & & 1.0000 \\
\hline Present & $20(80.0)$ & $5(20.0)$ & \\
\hline Absent & $32(78.0)$ & $9(22.0)$ & \\
\hline Extrathyroidal-extension & & & 1.0000 \\
\hline Present & $17(77.3)$ & $5(22.7)$ & \\
\hline Absent & $35(79.5)$ & $9(20.5)$ & \\
\hline
\end{tabular}

Table III. Associations between lymph node metastasis and clinicopathological parameters of papillary thyroid carcinomas.

\begin{tabular}{|c|c|c|c|}
\hline Parameter & Lymph node metastasis $+(\%)$ & Lymph node metastasis - (\%) & P-value \\
\hline No. of cases & $14(21.2)$ & $52(78.8)$ & \\
\hline Age (years) & $47.4 \pm 10.0$ & $50.0 \pm 10.4$ & 0.3964 \\
\hline Gender & & & 0.7441 \\
\hline Male & $4(23.5)$ & $13(76.5)$ & \\
\hline Female & $10(20.4)$ & $39(79.6)$ & \\
\hline Tumor size (mm) & $9.8 \pm 7.0$ & $8.4 \pm 6.7$ & 0.4881 \\
\hline Multiplicity & & & 0.2182 \\
\hline Present & $3(12.0)$ & $22(88.0)$ & \\
\hline Absent & $11(26.8)$ & $30(73.2)$ & \\
\hline Bilaterality & & & 0.0280 \\
\hline Present & $0(0.0)$ & $15(100.0)$ & \\
\hline Absent & $14(27.5)$ & $37(72.5)$ & \\
\hline Extrathyroidal-extension & & & 0.5244 \\
\hline Present & $6(27.3)$ & $16(72.7)$ & \\
\hline Absent & $8(18.2)$ & $36(81.8)$ & \\
\hline Lymphovascular-invasion & & & - \\
\hline Present & $0(0.0)$ & $0(0.0)$ & \\
\hline Absent & $14(21.2)$ & $52(78.8)$ & \\
\hline p53 overexpression & & & 0.4734 \\
\hline Present & $10(19.2)$ & $42(80.8)$ & \\
\hline Absent & $4(28.6)$ & $10(71.4)$ & \\
\hline BRAF mutation & & & 1.0000 \\
\hline Present & $11(22.0)$ & $39(78.0)$ & \\
\hline Absent & 3 (18.7) & $13(81.3)$ & \\
\hline
\end{tabular}

$49.5 \pm 10.4$ years. The mean tumor size was $9.1 \pm 6.8 \mathrm{~mm}$. Multiplicity and extrathyroidal extension were present in $19(38 \%)$ and $20(40 \%)$ out of 50 cases, respectively.

Among the 16 patients without the BRAF (V600E) mutation, 5 were males $(31.3 \%)$ and 11 were females $(68.7 \%)$, with a mean age of $49.3 \pm 10.4$ years. The mean tumor size was
$7.2 \pm 6.6 \mathrm{~mm}$. Multiplicity and extrathyroidal extension were present in $6(37.5 \%)$ and $2(12.5 \%)$ of the 16 cases, respectively (Table I).

As shown in Table I, none of the parameters exhibited a significant correlation with the BRAF (V600E) mutation $(\mathrm{P}>0.05)$; however, there was a notable, but 
Table IV. Associations between BRAF (V600E) mutation and p53 protein overexpression in papillary thyroid carcinomas.

\begin{tabular}{lccc}
\hline Parameter & BRAF mutation $+(\%)$ & BRAF mutation - (\%) & P-value \\
\hline No. of cases & $50(75.8)$ & $16(24.2)$ & 0.0854 \\
p53 overexpression & $42(80.8)$ & $10(19.2)$ & \\
Present & $8(57.1)$ & $6(42.9)$ & \\
Absent & &
\end{tabular}

non-significant, association between extrathyroidal extension and the BRAF (V600E) mutation $(\mathrm{P}=0.0661)$.

Associations between p53 protein overexpression and clinicopathological features of PTC. As shown in Table II, among the 52 patients who exhibited p53 protein overexpression, 14 were males $(26.9 \%)$ and 38 were females $(73.1 \%)$, with a mean age of $49.7 \pm 10.1$ years and a mean tumor size of $9.1 \pm 6.7 \mathrm{~mm}$. Multiplicity and extrathyroidal extension were present in $20(38.5 \%)$ and $17(32.7 \%)$ out of 52 cases, respectively.

Among the 14 patients who demonstrated no 553 protein overexpression, 3 were males $(21.4 \%)$ and 11 were females (78.6\%), with a mean age of $48.6 \pm 11.5$ years and a mean tumor size of $7.0 \pm 6.9 \mathrm{~mm}$. Multiplicity and extrathyroidal extension were each present in 5 out of 14 (35.7\%) cases (Table II).

As shown in Table II, none of the clinicopathological parameters demonstrated a significant correlation with p53 protein overexpression.

Associations between lymph node metastasis and clinicopathological parameters of PTC. As shown in Table II, among the 14 patients with lymph node metastasis, 4 were males $(28.6 \%)$ and 10 were females $(71.4 \%)$, with a mean age of $47.4 \pm 10.0$ years and a mean tumor size of $9.8 \pm 7.0 \mathrm{~mm}$. Multiplicity and bilaterality were present in $3(21.4 \%)$ and 0 of the 14 cases, respectively. Extrathyroidal extension and lymphovascular invasion were present in $6(42.9 \%)$ and 0 of the 14 cases, respectively. In addition, p53 overexpression and the BRAF (V600E) mutation were present in 10 (71.4\%) and $11(78.6 \%)$ of the cases, respectively.

As shown in Table III, only one parameter, bilaterality was demonstrated to be significantly associated with lymph node metastasis $(\mathrm{P}=0.0280)$.

Association between BRAF (V600E) mutation and p53 protein overexpression in PTC. Among the 50 patients with the BRAF (V600E) mutation, 42 patients exhibited p53 protein overexpression (84\%). In addition, among the 16 patients without BRAF (V600E) mutation, 10 patients demonstrated p53 protein overexpression (62.5\%) (Table IV). Therefore, there was a notable, but not a statistically significant, correlation between the BRAF (V600E) mutation and p53 protein overexpression $(\mathrm{P}=0.0854)$.

\section{Discussion}

BRAF is the most prevalent type of genetic alteration in thyroid cancer and has been widely investigated (27). In thyroid cancer, BRAF was reported to be activated via certain point mutations, including a nucleotide position 1799 substitution of thymine to adenine, which subsequently results in the replacement of valine with glutamate at residue 600 (V600E). Out of all the BRAF mutations that may occur in thyroid cancer, the BRAF (V600E) mutation accounts for $>90 \%$. The incidence rate of the BRAF (V600E) mutation varies greatly, ranging from 29 to $83 \%$ in PTC. The reason for this variation is unclear, but it is suggested that geographic, genetic factors, or other factors may account for these differences (9). In addition, it was reported that in Korean populations, the BRAF (V600E) mutation in PTC was markedly more prevalent at 52-83\% compared with $30-49 \%$ in other countries (9). It has not been determined why this variation occurs, although it was suggested that geographic or genetic factors may be involved (9). BRAF has been identified in classic papillary and tall cell thyroid cancer as well as in $\sim 1$ out of 3 cases of poorly differentiated and anaplastic thyroid carcinomas (27). The results of the present study determined the prevalence of the BRAF (V600E) mutation to be $75.8 \%$.

Numerous studies have evaluated the association between the BRAF (V600E) mutation, clinicopathological features and clinical outcomes of PTC; however, controversial results have been obtained for a correlation between the BRAF (V600E) mutation and clinicopathological features (1,9-20). Furthermore, multiple previous studies have demonstrated a correlation between the BRAF (V600E) mutation and the high-risk clinicopathological characteristics of PTC, including older age at diagnosis, male gender, large tumor size, the presence of extrathyroidal extension, lymph node and distant metastasis and an advanced stage (9-11,13, $15,16,18-20,28,29)$. By contrast, certain studies were unable to identify marked associations between the BRAF (V600E) mutation and the high-risk clinicopathological characteristics of PTC $(12,14)$. The reason for this variation is unclear, but it may be that geographic or genetic factors may account for these differences. However, the variation in these results is also considered to be the result of a lack of prospective studies that may reduce the selection bias, small study population sizes, the absence of multivariate analysis and the heterogeneous histological subtypes within PTC (9)

The results of the present study did not demonstrate any significant correlations between the BRAF (V600E) mutation and the clinicopathological features of PTC, including age, gender, tumor size, multiplicity, lymph node metastasis and extra thyroidal extension. However, extrathyroidal extension exhibited a borderline correlation with the BRAF (V600E) mutation $(\mathrm{P}=0.0661)$. 
The tumor suppressor gene p53 encodes a DNA-binding protein that has important functions in cell cycle arrest, DNA repair, differentiation and apoptosis. p53 gene mutations have been observed in $\sim 50 \%$ of the human cancers and are one of the most prevalent types of genetic modifications identified in malignant cells; in addition, these mutations primarily occur in exons $(3,4,5,7,8,23)$. In the thyroid gland, mutations of the p53 gene were reported to occur in $40-62 \%$ of undifferentiated carcinomas and $0-25 \%$ in well-differentiated carcinomas $(4,8)$. The overexpression of $\mathrm{p} 53$, as determined by immunohistochemistry was suggested to attributed to the mutation of a p53 gene in up to $95 \%$ of PTC cases (5). p53 protein overexpression was reported to have an incidence rate of between 11 and $59 \%(4,21,23)$.

Due to its short half-life, wild-type p53 protein is undetectable; however, mutated p53 exhibits greater stability and a prolonged half-life (24). Therefore, it was previously hypothesized that immunohistochemistry was only able to detect mutated p53. By contrast, it has been reported that the overexpression of p53 may not always be attributed to gene mutations, as wild-type overexpression may occur due to factors that have not yet been elucidated and may provide a protection mechanism against tumors $(5,23)$. In addition, mutations of p53 were proposed to induce the expression of abnormal proteins or lead to a deficient expression of p53 (23).

In thyroid cancers, it was suggested that the presence of p53, as detected by immunohistochemistry, was associated with the occurrence of p53 gene mutations (5); however, the detection of 553 protein expression was also reported in differentiated papillary and follicular thyroid carcinomas regardless of the occurrence of p53 gene mutations; of note, the incidence rate of $\mathrm{p} 53$ protein overexpression in PTC was reported to be between 11 and 59\% (4,21-25).

Certain studies regarding the immunohistochemical analysis of p53 expression have demonstrated that p53 overexpression may act as a significant and independent prognostic indicator for differentiated thyroid carcinomas $(3,4,7,22,24)$. However, this association has provided controversial results $(3,5,21,30,31)$. Morita et al (4) reported a significant correlation among p53 protein expression in primary tumors, larger tumors, the presence of lymph node metastasis and the mean number of lymph node metastases (4). Furthermore, Horie et al (7) indicated that p53 protein overexpression had a marked correlation with large tumor size and the occurrence of capsular invasion (7). However, several studies have reported no significant associations between p53-positive tumor cells and clinicopathological data $(5,6,21,30,31)$.

In the present study, no significant correlations were identified between 553 protein overexpression and clinicopathological features of PTC, including age, gender, tumor size, multiplicity, lymph node metastasis and extrathyroidal extension. Limitations of the present study included an insufficient number of prospective studies to reduce selection bias, a relatively small population size and the lack of multivariate analysis.

In conclusion, the results of the present study revealed that the BRAF (V600E) mutation and overexpression of p53 were not significantly correlated with clinicopathological features of PTC; however, the BRAF (V600E) mutation demonstrated a notable, but non-significant, association with p53 overexpression $(\mathrm{P}=0.0854)$ and extrathyroidal extension $(\mathrm{P}=0.0661)$. In addition, a significant correlation was observed between lymph node metastasis and bilaterality $(\mathrm{P}=0.0280)$. Further prospective studies are required, with a larger study population in order to determine the exact role of the BRAF (V600E) mutation and p53 protein overexpression in the clinicopathological significance of PTC.

\section{References}

1. Koperek O, Kornauth C, Capper D, Berghoff AS, Asari R, Niederle B, von Deimling A, Birner P and Preusser M: Immunohistochemical detection of the BRAF V600E-mutated protein in papillary thyroid carcinoma. Am J Surg Pathol 36: 844-850, 2012.

2. Zheng X, Wei S, Han Y, Li Y, Yu Y, Yun X, et al: Papillary microcarcinoma of the thyroid: Clinical characteristics and BRAF (V600E) mutational status of 977 cases. Ann Surg Oncol 20: 2266-2273, 2013.

3. Balta AZ, Filiz AI, Kurt Y, Sucullu I, Yucel E and Akin ML: Prognostic value of oncoprotein expressions in thyroid papillary carcinoma. Med Oncol 29: 734-741, 2012.

4. Morita N, Ikeda Y and Takami H: Clinical significance of p53 protein expression in papillary thyroid carcinoma. World $\mathbf{J}$ Surg 32: 2617-2622, 2008.

5. Zafon C, Obiols G, Castellvi J, Tallada N, Baena JA, Simo R, et al: Clinical significance of $\mathrm{RET} / \mathrm{PTC}$ and $\mathrm{p} 53$ protein expression in sporadic papillary thyroid carcinoma. Histopathology 50: 225-231, 2007 .

6. Hamzany Y, Soudry E, Strenov Y, Lipschitz N, Segal K, Hadar T, et al: Early death from papillary thyroid carcinoma. Am J Otolaryngol 33: 104-108, 2012.

7. Horie S, Maeta H, Endo K, Ueta T, Takashima K and Terada T: Overexpression of p53 protein and MDM2 in papillary carcinomas of the thyroid: Correlations with clinicopathologic features. Pathol Int 51: 11-15, 2001

8. Parameswaran R, Brooks S and Sadler GP: Molecular pathogenesis of follicular cell derived thyroid cancers. Int J Surg 8: 186-193, 2010

9. Kim SJ, Lee KE, Myong JP, Park JH, Jeon YK, Min HS, et al: BRAF V600E mutation is associated with tumor aggressiveness in papillary thyroid cancer. World J Surg 36: 310-317, 2012.

10. Kim KH, Kang DW, Kim SH, Seong IO and Kang DY: Mutations of the BRAF gene in papillary thyroid carcinoma in a Korean population. Yonsei Med J 45: 818-821, 2004.

11. Musholt TJ, Schonefeld S, Schwarz CH, Watzka FM, Musholt PB, Fottner $\mathrm{C}$, et al: Impact of pathognomonic genetic alterations on the prognosis of papillary thyroid carcinoma. ESES vienna presentation. Langenbecks Arch Surg 395: 877-883, 2010.

12. Ahn D, Park JS, Sohn JH, Kim JH, Park SK, Seo AN and Park JY: BRAF (V600E) mutation does not serve as a prognostic factor in Korean patients with papillary thyroid carcinoma. Auris Nasus Larynx 39: 198-203, 2012.

13. Ricarte-Filho J, Ganly I, Rivera M, Katabi N, Fu W, Shaha A, et al: Papillary thyroid carcinomas with cervical lymph node metastases can be stratified into clinically relevant prognostic categories using oncogenic BRAF, the number of nodal metastases and extra-nodal extension. Thyroid 22: 575-584, 2012.

14. Kurt B, Yalcin S, Alagoz E, Karslioğlu Y, Yigit N, Gunal A, et al: The relationship of the BRAF (V600E) Mutation and the established prognostic factors in papillary thyroid carcinomas. Endocr Pathol 23: 135-140, 2012.

15. Prescott JD, Sadow PM, Hodin RA, Le LP, Gaz RD, Randolph GW, et al: BRAF V600E status adds incremental value to current risk classification systems in predicting papillary thyroid carcinoma recurrence. Surgery 152: 984-990, 2012.

16. Elisei R, Viola D, Torregrossa L, Giannini R, Romei C, Ugolini C, et al: The BRAF (V600E) mutation is an independent, poor prognostic factor for the outcome of patients with low-risk intrathyroid papillary thyroid carcinoma: single-institution results from a large cohort study. J Clin Endocrinol Metab 97: 4390-4398, 2012.

17. Daglar-Aday A, Toptas B, Ozturk T, Seyhan F, Saygili N, Eronat A, et al: Investigation of BRAF V600E mutation in papillary thyroid carcinoma and tumor-surrounding nontumoral tissues. DNA Cell Biol 32: 13-18, 2013 
18. Jeong D, Jeong Y, Park JH, Han SW, Kim SY, Kim YJ, et al: BRAF (V600E) mutation analysis in papillary thyroid carcinomas by peptide nucleic acid clamp real-time PCR. Ann Surg Oncol 20 759-766, 2013.

19. Brahma B, Yulian ED, Ramli M, Setianingsih I, Gautama W, Brahma P, et al: Surgical perspective of T1799A BRAF mutation diagnostic value in papillary thyroid carcinoma. Asian Pac J Cancer Prev 14: 31-37, 2013.

20. Xing M, Alzahrani AS, Carson KA, Viola D, Elisei R, Bendlova B, et al: Association between BRAF V600E mutation and mortality in patients with papillary thyroid cancer. JAMA 309: 1493-1501, 2013.

21. Gauchotte G, Philippe C, Lacomme S, Leotard B, Wissler MP Allou L, et al: BRAF, p53 and SOX2 in anaplastic thyroid carcinoma: evidence for multistep carcinogenesis. Pathology 43: 447-452, 2011.

22. Park KY, Koh JM, Kim YI, Park HJ, Gong G, Hong SJ, et al: Prevalences of Gs alpha, ras, p53 mutations and ret/PTC rearrangement in differentiated thyroid tumours in a Korean population. Clin Endocrinol (Oxf) 49: 317-323, 1998.

23. Liu MC and Gelmann EP: P53 gene mutations: case study of a clinical marker for solid tumors. Semin Oncol 29: 246-257, 2002.

24. Omar E, Madhavan M and Othman NH: Immunohistochemical localisation of RET and p53 mutant protein of thyroid lesions in a North-Eastern Malaysian population and its prognostic implications. Pathology 36: 152-159, 2004.
25. Dellis RA, Lloyd RV, Heitz U and Eng C (eds): Tumours of endocrine organs. In: Pathology and Genetics. International Agency for Research on Cancer Press, Lyon, pp57-66, 2004.

26. Mitzner R: Neck Dissection Classification. http://emedicine. medscape.com/article/849834-overview. Accessed June 9th, 2015.

27. Witt RL, Ferris RL, Pribitkin EA, Sherman SI, Steward DL and Nikiforov YE: Diagnosis and management of differentiated thyroid cancer using molecular biology. Laryngoscope 123 : 1059-1064, 2013.

28. Min HS, Lee C and Jung KC: Correlation of immunohistochemical markers and BRAF mutation status with histological variants of papillary thyroid carcinoma in the Korean population. J Korean Med Sci 28: 534-541, 2013.

29. Smith RA, Salajegheh A, Weinstein S, Nassiri M and Lam AK: Correlation between BRAF mutation and the clinicopathological parameters in papillary thyroid carcinoma with particular reference to follicular variant. Hum Pathol 42: 500-506, 2011.

30. Cvejic D, Selemetjev S, Savin S, Paunovic I, Petrovic I and Tatic S: Apoptosis and proliferation related molecules (Bcl-2, Bax, p53, PCNA) in papillary microcarcinoma versus papillary carcinoma of the thyroid. Pathology 40: 475-480, 2008.

31. Karlidag T, Cobanoglu B, Keles E, Alpay HC, Ozercan I, Kaygusuz I, et al: Expression of Bax, p53 and p27/kip in patients with papillary thyroid carcinoma with or without cervical nodal metastasis. Am J Otolaryngol 28: 31-36, 2007. 